\title{
Phraseologismen in deutschen und polnischen Pressetexten, dargestellt aus pragmalinguistischer Perspektive
}

\author{
Janusz Pociask (Bromberg)
}

\begin{abstract}
The aim of this article is to present selected aspects of using some various idioms in texts of Polish and German press. Within the theoretical framework the following aspects are discussed: the constitutive features of idioms, their potential to build some texts, as well as appearing in particular texts. The analysis of press texts in both languages showed that idioms are a common link of expression and their adaptation and implementation are varied. Besides using canonical forms there are numerous idiom innovations involving the reduction, substitution and expansion in idiom components and breaking the phraseological structure, which results that they become a constitutive element of the text. What is important, all manipulations including actualization of form and meaning of the phraseological units are used for the purpose of text. They are not seen as a special use of lexical units but as typical treatments used in press, both in Polish and German. The article also discusses the features of idioms in press text.
\end{abstract}

\section{$1 \quad$ Ein paar Sätze vorweg}

Phraseologismen bildeten und bilden ein häufiges Untersuchungsobjekt. Anfänglich beschäftigte man sich ausführlich mit ihnen im Sprachsystem und versuchte, das Feld der phraseologischen Forschung abzustecken, die Terminologie, Definitionen und Bestimmungsmerkmale herauszuarbeiten sowie Klassifikationen aufzustellen.

Mit der pragmatischen Wende verschiebt sich die Forschung auf die Verwendung von Phraseologismen in unterschiedlichen kommunikativen Situationen. Man geht von der Textsortenspezifik aus und untersucht den Status der Phraseologismen in einzelnen Texten, ihre kommunikativ-pragmatischen Funktionen sowie die pragmatischen Möglichkeiten, die sich aus den vom System aus gegebenen Potenzen ergeben.

In der Forschung scheinen die Herausarbeitung von Definitionen, Klassifikationsvorschlägen und somit auch Beschreibungen von strukturellen Ausprägungen fester Phrasen abgeschlossen zu sein. In den entstehenden Diskussionen zur Phraseologie werden Klassifikationen, Terminologien den älteren Arbeiten ganz übernommen oder für die Zwecke der jeweiligen Untersuchung angepasst. Die pragmatischen Herangehensweisen an die Phraseologie erweisen sich dagegen als einer der wichtigsten Zugänge zur Phraseologie, zumal erst der Gebrauch der Phraseologismen ihre formale und semantische Struktur sowie ihre Funktionen tatsächlich erkennen lässt. Auf Grund der starken Abhängigkeiten fester Linguistik online 74, 5/15 - http://dx.doi.org/10.13092/lo.74.2227

CC by 3.0 
Phrasen von der textuellen Umgebung und der erwähnten Textsortenspezifik wird ersichtlich, wie aufschlussreich und erkenntnisgewinnend ihre Untersuchung im Rahmen der Pragmatik sein kann. ${ }^{1}$

Im Allgemeinen wird die linguistische Pragmatik als Lehre vom Gebrauch der sprachlichen Zeichen und Sprachhandlungen in konkreten Situationen und zu Kommunikationszwecken verstanden. Der Forschungsumfang der Pragmatik ist weiträumig und umfasst alle kontextabhängigen Aspekte der Sprache, dies betrifft Intentionen der Kommunikationspartner, Regularitäten und Rituale in der Kommunikation, Funktionen der sprachlichen Zeichen, aber auch das Repertoire der Sprachstrukturen in Kontexten (vgl. Filatkina 2007: 132).

Phraseologismen aus der pragmalinguistischen Perspektive zu untersuchen, heißt in erster Linie nach ihren kommunikativ-pragmatische Leistungen in Texten zu fragen. Im Mittelpunkt pragmatischer Aspekte stehen vor allem die Funktionen von Phraseologismen, die sich einerseits aus ihrer formalen und semantischen Beschaffenheit ergeben und die andererseits in der kontextabhängigen Entfaltung sichtbar zur Geltung kommen. Dies ist das Hauptanliegen der vorliegenden Überlegungen, die sich zugleich als Beitrag zur textphraseologischen Forschung verstehen. Dabei wird die Bedeutung der festen Phrasen in der Sprachverwendung betont und auf die Entfaltung der textbildenden Potenzen von Phraseologismen hingewiesen.

Die Möglichkeiten, die feste Phrasen im Text bieten, können für die Wirkung verschiedener Texte genutzt werden und legen damit auch die Potenziale von Phraseologismen offen.

Im Rahmen des Beitrags muss aus Platzgründen auf einen detaillierten Forschungsüberblick über textphraseologische Arbeiten verzichtet werden. ${ }^{2}$

Einleitend wird zuerst auf die Definition und Merkmale von Phraseologismen eingegangen. Diese kurze Diskussion dient zum einen dazu, den Forschungsgegenstand und seine Merkmale zu bestimmen und ist zum anderen als Ausgangsbasis für weitere Überlegungen zu textbildenden Potenzen von Phraseologismen und ihrer Textsortenspezifik notwendig. Anschließend folgt die Diskussion der pragmatischen Leistungen fester Phrasen in deutschen und polnischen Pressetexten. Im Laufe der Überlegungen wird auf einige Arbeiten verwiesen, die sich mit dieser Problematik auseinandergesetzt haben, wobei soweit möglich versucht wird, die dort gewonnenen Forschungsergebnisse nicht zu wiederholen, sondern ergänzende Erkenntnisse festzuhalten.

Der vorliegende Beitrag versteht sich als ein zusammenfassender Forschungsbericht. ${ }^{3} \mathrm{Im}$ Rahmen des Projekts wurde der Verwendung von Phraseologismen in unterschiedlichen

\footnotetext{
${ }^{1}$ Solche Untersuchungen können sowohl für die Phraseographie, kontrastive Textphraseologie, Übersetzungspraxis, Phraseodidaktik als auch für die Rhetorik und Stilistik von Nutzen sein.

2 An dieser Stelle wird auf die Studienbibliografien zur phraseologischen Fachliteratur von Földes (1997) oder Donalies (2012) verwiesen. Bei Fleischer (1997: 261-266) und Pociask (2007a: 14-17) finden sich ebenfalls Hinweise und Angaben zu Untersuchungen der festen Wortverbindungen in Texten. Mit Phraseologie. Ein internationales Handbuch der zeitgenössischen Forschung (2007) liegt ein umfassendes Werk zur Phraseologie vor, das auch einige pragmalinguistische Aspekte ins Auge fasst. Den einzelnen Beiträgen ist ebenfalls eine umfangreiche Literatur in Auswahl zum weiteren Studium zu entnehmen.

${ }^{3}$ Der Zeitraum der Untersuchung erstreckt sich von 2004 bis 2007 (Untersuchung deutscher Pressetexte) und von 2012 bis 2014 (Untersuchung von deutschen und polnischen Pressetexten).
}

ISSN 1615-3014 
dargestellt aus pragmalinguistischer Perspektive

Texten nachgegangen. Die Erkenntnisse beziehen sich hier auf polnische und deutsche Pressetexte. Den Überlegungen liegt ein umfangreiches Korpus zugrunde, das zum einem aus der Neuen Zürcher Zeitung im Zeitraum 2003-2004 und zum anderen aus weiteren deutschen und polnischen Zeitungen und Zeitschriften zwischen Oktober 2009 bis Juni 2014 entnommen wurde. ${ }^{4}$ Ich untersuche den Gebrauch von Phraseologismen in informierenden (Meldungen und Berichten, Reportagen) und meinungsäußernden (Kommentaren und Glossen) Darstellungsformen, unabhängig von der thematischen Orientierung der Texte.

Das Korpus umfasst insgesamt 3609 Belege, davon 3367 aus der Neuen Zürcher Zeitung, und 180 Beispiele aus den restlichen deutschen und 162 aus polnischen Quellen. Alle Aussagen im vorliegenden Beitrag resultieren aus der Analyse der Korpusbeispiele. ${ }^{5}$

Die pragmatische Perspektive, die hier diskutiert wird, ist nur einer der Aspekte, denen im Rahmen des Projekts nachgegangen wird.

\section{Definition, Terminologie und Wesensmerkmale}

Die Begriffsfestlegung von Phraseologismus ist in vielen Arbeiten eine Ausgangsfrage. Dabei werden unterschiedliche Auffassungen vertreten, und das Bestreben, alle mehr und minder festen Phrasen exakt zu definieren und möglichst lückenlos zu klassifizieren, führt oft zu sehr ausgebauten Definitionen und Klassifikationen von festen Phrasen. ${ }^{6}$ Dies zeigt, wie problematisch, wenn überhaupt möglich, eine eindeutige Abgrenzung fester Phrasen untereinander und von angrenzenden sprachlichen Phänomenen ist.

Phraseologismen seien hier in Anlehnung an die Definition von Burger et al. (1982: 1) und die von Fleischer (1997: 29-30) aufgefasst als mehrgliedrige, stabile, im Phraseolexikon gespeicherte und reproduzierbare Verbindungen von Wörtern, deren Gesamtbedeutung nicht oder nur teilweise aus der Bedeutung der einzelnen Wörter ableitbar ist. ${ }^{7}$ Aus der Definition ergeben sich folgende Kriterien für die Bestimmung von festen Wortverbindungen: Polylexikalität, Stabilität, Lexikalisierung, Reproduzierbarkeit und Idiomatizität. Während die Polylexikalität als formales Erkennungsmerkmal unumstritten ist, ${ }^{8}$ müssen andere Kriterien, vor allem die formal-semantische Stabilität, relativiert werden. Zwar kann man die Komponenten bei nicht wenigen Phraseologismen "auf der syntagmatischen Ebene nicht frei kombinieren und auf der paradigmatischen Ebene nicht frei substituieren" (Palm 1995: 7), sodass sie in ihrer Verwendung im Gegensatz zu freien Wortgruppen begrenzt sind, was oft

\footnotetext{
${ }^{4}$ Der Spiegel, Der Stern, Handelsblatt, Süddeutsche Zeitung (SZ), Gazeta Wyborcza (GW), Polityka, Wprost und Newsweek. Hier wurde absichtlich nicht auf jeweils nur eine Quelle zurückgegriffen, um einen Gesamteindruck zu bekommen. Zwar werden Aussagen zur Textsortenspezifik von Phraseologismen gemacht, bei der Auswahl der Texte wurde jedoch nicht auf ihre Zugehörigkeit zu einer bestimmten Textsorte geachtet.

${ }^{5}$ Aus Platzgründen und der Übersichtlichkeit halber werden im Beitrag ausgewählte Beispiele dargeboten. Oft erscheinen sie im Laufe des Textes in der verkürzten Form, immer mit der Angabe der Quelle.

${ }^{6}$ Auf die Klassifikations-, Abgrenzungs- von Definitionsprobleme wird hier zum einen aus Platzgründen und zum anderen deswegen, dass dies in der Forschung häufig Gegenstand der Diskussion und bereits gesichert ist, verzichtet. Verwiesen wird z. B. auf Burger at al. (1982), Fleischer (1997) oder Phraseologie. Ein internationales Handbuch der zeitgenössischen Forschung (2007). Diese Arbeiten gelten als Standardwerke und Grundlagen für weitere diesbezügliche Diskussionen.

${ }^{7}$ Vgl. die Überlegungen zur Bedeutung von Phraseologismen von Pajdzińska (2001: 11).

${ }^{8}$ Obwohl sie nicht selten Probleme für die lexikographische Erfassung darstellt.
} 
durch unikale Komponenten oder grammatische Restriktionen bzw. durch sog. transformationelle Defektivität (vgl. Fleischer 1997: 49-56) verstärkt wird, aber es lassen sich bei vielen festen Wortverbindungen Varianten oder kontextuelle Abwandlungen beobachten. Daher ist die Stabilität nicht als absolutes Kriterium zu betrachten. Sie besteht m. E. darin, dass jeder einzelnen usuellen oder ungewöhnlich gebrauchten Form, jeder mehr oder weniger auffälligen Abwandlung bzw. textuellen Aktualisierung eine reproduzierbare Form zugrunde liegt (vgl. Pociask 2007a: 22). Daher weisen Phraseologismen unterschiedliche Grade der Stabilität und ihre Komponenten unterschiedliche Aktivität im Text auf, weil die Komponenten trotz ihrer semantischen Umdeutung im Phraseologismus eine relative Autonomie bewahren (vgl. Hessky 1992: 83).

Die Tatsache, dass sie aus selbständigen Lexemen bestehen, die wiederum als eine neue selbständige lexematische Verbindung von Wörtern fungieren, macht sie zu komplexen Einheiten. Ihre Komplexität gegenüber den Einzellexemen folgt weniger aus ihrer ganzheitlichen (phraseologischen) Bedeutung, sondern aus der mehrgliedrigen Struktur und der kollokativen Zugehörigkeit der Komponenten, die sich aus der psycholinguistischen Perspektive erschließen lässt. Darüber hinaus macht sich die Polylexikalität, die als das einzige Bestimmungskriterium unproblematisch zu sein scheint und Phraseologismen von Lexemen abhebt, in ihrer Semantik geltend.

Die Wesensmerkmale, die Phraseologismen im Sprachsystem charakterisieren, sind als idealisierte Mustermerkmale zu betrachten und daher zu relativieren. Die Beobachtung des Sprachgebrauchs zeigt auf, dass feste Phrasen in Texten häufig in einer formal-semantischen Aktualisierung bzw. Abweichung unterschiedlichen Grades auftreten. Die Abweichung kann von einer kontextuellen (formalen) Aktualisierung der (meist verbalen) Komponenten oder einer Substitution, Reduktion oder Expansion im Bereich der phraseologischen Komponenten bis hin zur kontextabhängigen Verfremdung und völligen Auflösung der phraseologischen Struktur reichen. ${ }^{9}$ Den Verwendungsmöglichkeiten sind ausschließlich funktionale Beschränkungen auferlegt. Daher gilt der Aufruf von Gréciano (1983: 238): Um das Wesen der Phraseologismen adäquat $\mathrm{zu}$ beschreiben, ist eine Hinwendung zum Sprachgebrauch unerlässlich.

\section{Textbildende Potenzen von Phraseologismen}

Wotjak (1992: 3) hebt zu Recht hervor, dass Phraseologismen als systemhaft relativ stabile, polylexikale Einheiten durch - neben den oben erwähnten Merkmalen - "eine hohe, in der Rede aktualisierbare textbildende (kreative Verknüpfungs-, Assoziations- und Modifikations-) Potenz, ein sehr reiches kommunikatives Potential" gekennzeichnet sind. Dieses Potenzial ergibt sich daraus, dass es sich bei den Phraseologismen zwar um feste formal-semantische ganzheitliche Einheiten mehrgliedrigen Charakters handelt, die aber für den jeweiligen Kontext und je nach kommunikativer Situation ihre Merkmale aufheben können und vielfältige Möglichkeiten der textuellen Aktualisierung bieten. Darüber hinaus ist dies begünstigt durch ihre semantische und syntaktische Struktur, genau genommen durch die potenzielle Variabilität und Teilbarkeit vieler Phraseologismen, was wiederum auf die weitgehende Selbständigkeit der einzelnen Konstituenten zurückzuführen ist.

\footnotetext{
${ }^{9}$ Lewicki (2007: 9) hebt besonders auf den dynamischen Charakter fester Wortverbindungen hervor.
} 
Zwar lässt der textorientierte und pragmatisch-funktionale Zugang zur Phraseologie andere Erkenntnisinteressen in den Vordergrund rücken, er bedeutet jedoch nicht den absoluten Bruch mit den vom Sprachsystem aus erarbeiteten Forschungsergebnisse zur Phraseologie. In diesem Zusammenhang taucht der oft diskutierte Begriff der textbildenden Potenzen fester Phrasen auf. Wotjak (1992: 99) verweist dabei auf die Dialektik des Begriffs bei Dobrovol'skij (1980), dessen Bedeutung darin besteht, dass er gerade den systembezogenen, den kommunikativen und den textuellen Aspekt verbindet (vgl. Dobrovol'skij 1980: 690). Dobrovol'skij (1980: 691) definiert die textbildenden Potenzen als ein Resultat der Abstraktion, die aus der Analyse konkreter Texte und der Untersuchung der systemsprachlichen Spezifika der Phraseologismen gewonnen wird. Textbildende Potenzen werden demnach aus dem Verhältnis der systembezogenen Aspekte und der textuellen Aktualität bestimmt. Der Terminus 'Potenz' inkludiert, dass es sich hier um eine Möglichkeit handelt, Texte zu konstituieren, denn die Aktualisierung und Entfaltung der jeweiligen Potenz hängt vom Gesamtkontext und vielen Kommunikationsfaktoren ab, sodass man nur in einem konkreten Kontext erkennen kann, welche der textbildenden Potenzen aktiviert wird.

Die Potenzialität wird an jeder Eigenschaft des Phraseologismus ersichtlich. Strukturelle bzw. semantische Festigkeit der Einheit z. B. lassen formale und semantische Variabilitäten im Text zu. Auch unter pragmatischem Aspekt kann ein Phraseologismus seine potenziellen Funktionen je nach Text und vor allem Textfunktion realisieren. Phraseologismen verleihen den Texten mit ihren semantischen und stilistischen Eigenschaften und mit der Aufgabe, nicht die Benennungslücken zu schließen, sondern in der Kommunikation als expressive Konkurrenzformen zu bereits existierenden, nichtexpressiven Benennungen zu fungieren (vgl. Barz 1986: 321), Bildlichkeit und Expressivität. Die Potenzialität der Bedeutung kann ebenfalls auf der Textebene explizit gemacht werden. ${ }^{10}$ Auch die Polylexikalität als formale Eigenschaft von Phraseologismen kann eine textorganisierende und -verflechtende Funktion übernehmen, die Textvernetzung sichern sowie zur Verknüpfung von Textteilen und somit zur Textkohäsion und Organisation der Textstruktur beitragen. ${ }^{11}$ Als textbildendes Potenzial ist auch die Tatsache zu bewerten, dass Phraseologismen in den mehr oder weniger typischen Positionen im Text vorkommen können. Es sei selbstverständlich hervorgehoben, dass die Mechanismen der Textkonstitution, die sich aus der Mehrgliedrigkeit ergeben, zum semantischen Mehrwert des Textes beitragen können.

Gréciano (1987: 196) relativiert die Wesensmerkmale der Phraseologismen, indem sie Folgendes festhält: "Die Polylexikalität ist ein Appell an die Fragmentierung, die Fixiertheit an die Variabilität und die Figuriertheit an die Literalisierung". Die Beobachtungen des Sprachgebrauchs bestätigen, dass die bereits erwähnten Merkmale keine absoluten Größen darstellen, sondern als Variablen zu verstehen sind. Gerade das macht die Phraseologismen mit ihrer formalen und semantischen Varianz zu besonderen anpassungsfähigen Bausteinen der Texte. Interessant ist auch die Tatsache, dass diese Varianz ein wichtiges Wesensmerkmal

\footnotetext{
${ }^{10} \mathrm{Zu}$ denken ist hier an die semantischen Prozesse wie Demotivierung, Remotivierung oder Ambiguierung, die bei kreativer Verwendung eine wesentliche Rolle spielen und durch den Kontext und seine Elemente gesteuert werden (s. Beispiele 15-16, 24, 28-36). Vgl. dazu Lewicki (2007: 10) derywacje frazeologiczne.

${ }^{11}$ Als Beispiele dienen hier die textkonstitutiven Mechanismen wie Rahmenbildung, Juxtaposition, Spreizstellung, Extension und Phraseoschablonen bei Palm (1995: 66-68) oder formale Modifikationen der Abtrennung und Koordinierung (vgl. Pociask 2007a: 113-118).
} 
von festen Phrasen ist, das sie von allen sprachlichen Einheiten abhebt. ${ }^{12}$ Darüber hinaus sind alle formal-semantischen Abweichungen nur durch die prinzipielle Stabilität polylexikaler Form und ganzheitlicher Bedeutung möglich (vgl. Hessky 1992: 83).

\section{Textsortenspezifik der Phraseologismen am Beispiel der Pressetextsorten}

Unter Textsortenspezifik ist $\mathrm{zu}$ verstehen, dass Phraseologismen in bestimmten Texten häufiger als in den anderen vorkommen und dort besonderen Anteil an der Textkonstitution haben. Diese Eigenschaft wird in erster Linie auf Grund der Häufigkeit ihres Vorkommens in einer Textsorte, aber auch auf Grund der Art und Weise der Verwendung und ferner der kommunikativen Funktion von Phraseologismen in dem jeweiligen Text bestimmt. Burger at al. (1982: 152) haben auf Grund der Verwendungsweise und Bevorzugung bestimmter phraseologischer Einheiten auf ein klares differenziertes Merkmal von Texttypen hingewiesen. Die textsortentypische Frequenz und die Art der Verwendung von festen Phrasen unterschiedlicher Typen können den Rezipienten als einer der Indikatoren für journalistische Textsorten dienen. ${ }^{13}$

Will man die Phraseologismen in Pressetexten untersuchen, so stellt sich das Problem der Vielfalt journalistischer Texte. In jeder Zeitung/Zeitschrift ist eine Mischung von Texten zu finden. Die einzelnen Pressetexte sind Ressorts/Themen/thematischen Bereichen bzw. Blöcken/Rubriken etc. zugeordnet. ${ }^{14}$ Sie können ferner nach der Hauptfunktion (Intention, Absicht) in Textklassen und zugehörigen Textsorten, die konventionell geltende Muster für komplexe Sprachhandlungen darstellen, bestimmt werden. Lüger (1995: 65) unterscheidet folgende Textklassen: informationsbetonte, meinungsbetonte, auffordernde, instruierendanweisende und kontaktorientierte Texte. $\mathrm{Zu}$ den häufigsten journalistischen Textsorten ${ }^{15}$ gehören Meldungen, Berichte, Reportagen (informierende Darstellungsformen) und Kommentare und Glossen (meinungsäußernde Pressetexte). Die Pressetexte sind wie erwähnt keineswegs homogen. ${ }^{16}$ Die Inhomogenität ergibt sich aus den Unterschieden in der thematischen Orientierung, Semantik und Struktur der Pressetexte, ihren Funktionen sowie in der Wahl der sprachlichen Mittel. An dieser Stelle seien sprachliche textsortenspezifische

\footnotetext{
12 Lewicki (2007: 9) nennt Bereiche und Möglichkeiten der Veränderungen von Phraseologismen: paradygmat związku frazeologicznego, szereg wariacyjny i derywację związków frazeologicznych.

13 Allerdings ist es fraglich, ob sich die Rezipienten bei der Wahl der gelesenen Texte an Textsorten orientieren. Vielmehr sind es die Textthemen.

${ }^{14}$ Diese Zuordnung trotz detaillierter Differenzierung in Subthemen (z. B. Weltwirtschaft) scheint nicht unproblematisch, weil eine eindeutige thematische Zuweisung der Texte oft nicht möglich ist und daher für den Leser eine erste thematische Orientierung darstellt.

15 Textsorten "lassen sich als jeweils typische Verbindungen von kontextuellen (situativen), kommunikativfunktionalen und strukturellen (grammatischen und thematischen) Merkmalen beschreiben. Sie haben sich in der Sprachgemeinschaft historisch entwickelt und gehören zum Alltagswissen der Sprachteilhaber; sie besitzen eine normierende Wirkung, erleichtern aber zugleich den kommunikativen Umgang, indem sie den Kommunizierenden mehr oder weniger feste Orientierungen für die Produktion und Rezeption von Texten geben" (Brinker 2005: 144).

16 Obwohl bestimmte Textmuster und Darstellungsstrategien in der Presse Standards unterliegen, ist eine Tendenz zur Vermischung von journalistischen Textsorten zu beobachten. Majkowska (2001: 25) kommentiert dieses Phänomen wie folgt: "Obserwowana w mediach tendencja do zacierania się granic między gatunkami dziennikarskimi - ich przekształcanie w infotainment - skrzyżowanie informacji z rozrywką - sprzyja językowej inwencji dziennikarzy $[\ldots] "$.
} 
Elemente wie Phraseologismen mit ihrer textkonstitutiven Beschaffenheit zu nennen. In diesem Zusammenhang verweist Fleischer (1997: 223) auf solche Eigenschaften fester Phrasen wie semantische und syntaktische Teilbarkeit und Expressivität, Anschaulichkeit und Bildlichkeit. Ihre Textsortenspezifik ist in Bezug auf Pressetexte mehrmals nachgewiesen worden. Auch diese Untersuchung bestätigt das Phänomen. Zum einen ist im Allgemeinen ein auffällig häufiges Vorkommen der Phraseologismen verschiedener Klassen in der Presse zu beobachten und zum anderen gibt es unter den journalistischen Darstellungsformen in Bezug auf die Verwendung von Phraseologismen große textsortenspezifische Unterschiede, die auch trotz der prinzipiellen Unmarkiertheit der Phraseologismen in dieser Hinsicht (vgl. Fleischer 1997: 222) mit den unterschiedlichen pragmatischen Funktionen und den bereits erwähnten kommunikativen Eigenschaften zusammenhängen.

\section{$5 \quad$ Usuelle vs. auffällige Aktivierung der Phaseologismen}

Im Prozess der Textproduktion werden sprachliche Einheiten in ihrer Form und Bedeutung 'aktiviert'. Texte als pragmatische Größen verstanden (vgl. Gréciano 1987: 196) bieten Freiraum zur Entfaltung des phraseologischen Potenzials, das je nach Text unterschiedlich zum Ausdruck kommt. Die Aktivierung der textkonstitutiven Potenzen muss jedoch in bestimmten Rahmen erfolgen, damit die Kohäsion und dadurch die Kohärenz des Textes nicht beeinträchtigt werden. Phraseologismen werden in die textuelle Ganzheit eingebettet und unterliegen in erster Linie grammatischen Veränderungen an den Komponenten (etw. in den Wind schlagen, być komuś solą w oku).

(1) Und so macht North Carolina weiter wie bisher und schlägt die Warnungen der Wissenschaftler in den Wind. (Spiegel 50/2013)

(2) Rozpatrujac za i przeciw nie da się ukryć, że Romowie od dawna byli sola w oku mieszkańców osiedla (Wprost 25/2013)

Grundsätzlich ist zwischen Aktualisierungen usueller Art und phraseologischen Bildungen okkasionell-textgebundener auffälliger Art zu differenzieren. Hierzu zu zählen sind einerseits alle usuellen Varianten mit ihren Flexionsformen (vgl. Pociask 2010), die durch die grammatische Anpassung im Text entstehen, und andererseits alle Abweichungen (formale und semantische Auffälligkeiten - s. Beispiele im Beitrag) vom phraseologischen Usus. ${ }^{17}$ Textphraseologische Untersuchungen ergeben, dass dabei auf Grund der Quantität solcher Verwendung kein abweichender Sprachgebrauch vorliegt, sondern eine typische Erscheinung der Verwendung von Phraseologismen in Pressetexten (vgl. Barz 1986: 322). Neben solchen pragmatisch-semantischen Gründen wie Erhöhung des Rezeptionsanreizes, Verstärkung von Aussagen, Informationsverdichtung, Erreichung eines bestimmten wortspielerischen Effekts, Anschaulichkeit, Expressivität oder auch Nomination (s. dazu Barz 1986: 322 und Wotjak 1992: 101) kann sich der häufig auffällig-kreative Gebrauch daraus ergeben, dass die Expressivität der stilistisch markierten Phraseologismen "einem Verschleiß, einer Abnutzung unterliegt und dadurch eine ständige Tendenz zur Schaffung neuer expressiver Benennungen

\footnotetext{
${ }^{17}$ Dazu vgl. Diskussionen der phraseologischen Norm bei Burger (1987b), Bąba (1989), Elspaß (2002), Mosiołek-Kłosińska (2002), Fliciński (2011).
} 
wirksam ist" (Barz 1986: 322). Ersichtlich wird es vor allem an phraseologischen Kontaminationen und Substitutionen der festen Phrasen:

(3) Der stechende Dorn im Auge (Der Stern 3/2009)

(4) Amerykańska masakra tarcza antyrakietowa (Wprost 39/2009) ${ }^{18}$

Die kreative Verwendung von Phraseologismen kann sowohl auf der formalen als auch semantischen Ebene ansetzen, wobei man betonen muss, dass dabei insofern ein Zusammenhang besteht, als dass die Form auch semantische Veränderungen zur Folge hat (Ross 2001: 241). Daraus lässt sich folgern, dass semantische Auffälligkeiten sowohl durch den Kontext als auch durch auffällige Aktualisierungen der formalen Struktur entstehen können:

(5) Seit langem schon hat man dort den politischen Braten gerochen. (Der Stern 27/2013)

(6) Wygląda na to, że wszystkie możliwe klamki już zapadty. (GW 49/2013)

Bei der Bestimmung der formalen Aktualisierung geht es um die Art des 'Eingriffs' in die Struktur des Phraseologismus. Dabei wird, je nachdem welchen Veränderungen der wendungsinterne Komponentenbestand unterworfen wird, zwischen Substitution (7) den Boden unter den Füßen vermissen (Der Spiegel 37/2012), (8) od zarania transformacji (Wprost 22/2014), Expansion (9) noch mehr Öl ins politische Feuer (Der Spiegel 50/2013), Reduktion (10) Powrót na tono (Wprost 6/2014), Kontamination (3), grammatischen Aktualisierungen und ferner vielfältigen Kombinationen unterschieden: (11) Die Weste des HSI ist schon arg befleckt (NZZ 196/2003), (12) uciszacz największych brudów (Wprost 22/2014). Eine Sonderstellung unter den formalen Aktualisierungen nimmt die Abtrennung (13) der eigene Schatten, über den man zu springen versucht (Der Stern 14/2013) und die Koordinierung identischer Elemente von Phraseologismen ein: (14) Statt sich die Hände wund und schmutzig zu machen (SZ 5/2013) ein. ${ }^{19}$ Diese kontextuellen Aktualisierungen haben vielfältige textkonstitutive und pragmatische Funktionen. Die substituierten oder erweiterten Phraseologismen tragen zur Herstellung und Verstärkung der Kohärenz des Textes bei. Der textkonstitutive Effekt besteht ferner darin, dass die aktivierte phraseologische Form an der Themenentfaltung teilnimmt. Durch den Ersatz oder die Erweiterung im Komponentenbestand werden in die Wendung neue Elemente eingebracht (Beispiele 4, 5), die den Phraseologismus an den Kontext (in dem Fall 'Politik') semantisch anpassen. Dadurch gewinnt der Text an inhaltlicher Geschlossenheit. Gerade mit textgebundenen Substitutionen $(5,15)$ und Expansionen (16) wird die Spezifizierung und Konkretisierung angestrebt. Die phraseologische Basis bleibt erhalten und wird zusätzlich um neue kontextrelevante Elemente bereichert, die konkretisierend und spezifizierend (13) die Gefahr eines Krieges an die Wand malen (SZ vom 29.11.2013) wirken.

\footnotetext{
${ }^{18}$ Umwandlung eines Filmtitels Meksykanska masakra pita mechaniczna, der zu einem Geflügelten Wort wurde.

${ }^{19}$ In diesem Rahmen können die einzelnen formalen und semantischen Gebrauchsauffälligkeiten der Phraseologismen ausführlicher nicht diskutiert werden. Vgl. dazu Wotjak (1992: 133-153), Fleischer (1997: 207-212), Burger (1998: 150-154), Błachut (2004: 74-165), Pociask (2007a: 69-123).
} 
(15) Nun sollen die Journalisten aufhören, um den heißen Brei herumzuschreiben. (SZ vom 29.11.2013)

(16) Gdyby nie te nałogi, być może bylby w stanie wspiać się na szczyt politycznej drabiny (Wprost 22/2014)

Das kontextbedingte Weglassen der Komponente $(17,18)$, das besonders im Titelbereich (95\% aller Reduktionen) zu finden ist, stellt eine Art Rätsel dar, das im Haupttext gelöst wird und beim Textrezipienten eine hohe Sprachkompetenz voraussetzt. Durch die Kürze des Ausdrucks wird auf Grund des Kontrastes mit der Grundform des Phraseologismus erhöhte Expressivität erreicht.

In der Höhle des Löwen (SZ vom 29.11.2013)

$$
\text { Damoklesschwert (Der Spiegel 35/2009) }
$$

Olimpiada i czeski film (Wprost 7/2014) 20

Kolega koledze wilkiem (Polityka 4/2012)

Auch andere auffällige Aktualisierungen können der Informationsverdichtung und Textverflechtung dienen. Durch Attribuierung (21) von der Hand in den Mund lebenden Menschen müssen den Gürtel noch enger schnallen (SZ vom 29.11.2013), (22) wysoko postawiona poprzeczka (Wprost 9/2014) und Nominalisierung (23) Sprung über den eigenen Schatten (Der Spiegel 49/2013) oder Kontamination (3) entsteht text- und informationsverdichtende Wirkung. Die Funktion liegt in der Intensivierung und Emotionalisierung der Ausdrucksweise: (24) Panowie myjemy ręce (Wprost 7/2014). ${ }^{21}$

Die Abtrennung der Komponenten - z. B. durch einen Relativsatz - hat eine konkretisierende bzw. spezifizierende Aufgabe, da neue Informationen in den Text eingebracht werden: (25) Wolf, der sich einen Schafspelz umgehängt hat (NZZ 130/2003). Darüber hinaus bilden die abgetrennten Komponenten eine Art Rahmen, in dem die wendungsexternen Elemente des Phraseologismus und fakultative bzw. obligatorische externe Teile stehen und textorganisierend wirken. Dieser Rahmen und ferner die Wiederaufnahme der Komponenten in einem Text machen die Aktivierung der textkonstitutiven und dadurch kohärenzerzeugenden Potenzen der Phraseologismen ersichtlich. Die kontextuellen Aktualisierungen werden als Teil des Kontextes sowohl strukturell als auch semantisch in den Phraseologismus integriert, sodass sie mit dem Kontext fest verflochten sind.

Auf Grund semantischer Einschränkungen vieler Bildungen und Verknüpfungen (vgl. Fleischer 1997: 53) werden mit auffälligen Aktualisierungen humoristische Wirkungen und Überraschungseffekte angestrebt.

(26) Ein Mann in existenzieller Not, das Wasser steht ihm bis zum Hals, gleich wird er untertauchen. (NZZ 66/2003)

(27) Aber es sind Frauen und Mütter, die zuhören können und verstehen, wenn einer Frau die Decke auf den Kopf fällt - auch ausserhalb der Bürozeit (NZZ 94/2003)

\footnotetext{
${ }^{20}$ Im Lead des Textes: Igrzyska rozpoczęte, ale ich gospodarze ciagle na trasie sprintu budowlanego.

${ }^{21}$ Rahmentitels des Feuilletons: Zmatpychwstanie
} 
(28) Ptak rozwija skrzydta (Wprost 18/2014)22

(29) Mocne plecy w szkole (Wprost 34/2013)23

Der Effekt beruht auf der Einbringung zusätzlicher Informationen und verdichtender, unerwarteter Kombination und ungewöhnlicher Aktualisierungen sprachlicher Mittel. Um diese Funktionen zu erfüllen, müssen die aktualisierten Strukturen die ursprüngliche nicht umgewandelte Form erkennen lassen. Die verbliebenen Komponenten stellen den phraseologischen Kern dar, der als formale 'Kurzform' bzw. 'Vertreter' für den Phraseologismus steht und seine Bedeutung erschließen lässt. Wotjak (1992: 146) weist in Anlehnung an Burger at al. (1982: 195) darauf hin, dass "die im Text aktualisierten Bruchstücke eine Art Etikett oder Symbol für den dahinterstehenden Phraseologismus als Ganzes" sind (s. Beispiele 4, 18, 20). Der phraseologische Kern setzt auch formale und semantische Grenzen, wie weit die Veränderungen gehen können. Das Prinzip der kreativen Verwendung beruht darauf, dass das System bestimmte Rahmen für Veränderungen vorgibt, in denen eine Aktualisierung zugelassen oder blockiert wird. Diese vorgegebenen Rahmen für Aktualisierungen werden im Dienste der kreativen Verwendung gesprengt. Gerade die Eingrenzungen durch das System seien ein 'Appell' an die Kreativität des Textproduzenten (vgl. Gréciano 1987: 196).

Wotjak (1999: 57) bemerkt mit Recht, dass "das Vermögen der Phraseologismen zu verschiedenen kontextbedingten Aktualisierungen einer oder mehrerer ihrer Bedeutungen (Polysemie, Homonymie), zu formalen Abwandlungen und inhaltlichen Anspielungen in ihrer Eigenart liegt". Die kreative Verwendung der Phraseologismen auf der Inhaltsebene beruht in erster Linie auf "auffälligen Bedeutungsaktualisierungen" (Wotjak 1992: 109). Dazu gehören kontextuelle Aktualisierung der phraseologischen Bedeutung (Demotivierung - 30, 31, 32), Aktualisierung der Bedeutung der einzelnen Komponenten (Remotivierung - 33, 34) und Aktualisierung sowohl der phraseologischen Bedeutung als auch der wörtlichen Bedeutung der freien Wortgruppe (Ambiguierung - 35, 36).

(30) Merkels ganze Art zielt darauf ab, den Streitraum klein zu halten. Leise Sohlen und Samthandschuhe, das ist ihre Grundausstattung für die Politik. (Der Spiegel 50/2013)

(31) To tylko wierzchotek góry lodowej. (Wprost 3/2014)

(32) Oczekiwana zmian miejsc (Wprost 9/2014) 24

(33) Benjamin Winter saß über Jahre fest im Sattel. (SZ 16.06.2014)25

(34) Wielu z nich chciałoby stanać ponownie na nogi, choćby na jedna (Polityka $2 / 2012)^{26}$

\footnotetext{
22 Ptak ist ein Familienname eines Unternehmers.

${ }^{23}$ Es geht um Haltungsfehler bei Schulkindern.

24 Titel des Beitrags W imię ojca i syna. Im Lead des Textes: Następcy wychodza z cienia rodziców.

${ }^{25}$ Der Text handelt von einem tödlichen Unfall bei einem Reitturnier: Der Reiter ist bei einem Sturz ums Leben gekommen.

${ }^{26}$ Bericht über Opfer von Unfällen.
} 
Janusz Pociask: Phraseologismen in deutschen und polnischen Pressetexten, dargestellt aus pragmalinguistischer Perspektive

Die Wurst als Kommunikationsmittel im Wahlkampf. (...) Es geht hier sprichwörtlich um die politische Wurst. (Handelsblatt vom 20.09.2013)

(36) Szczyt ryzyka (Wprost 3/2014) ${ }^{27}$

Ferner zähle ich zur kreativen Verwendung der Phraseologismen den semantischen Überraschungseffekt, der auf einer inhaltlichen und formalen überraschenden Verknüpfung beruht ${ }^{28}$ :

(37) Im Klartext: Wasch mir umweltbewusst den Steuer-Pelz, aber mach mir ja kein Diesel-Auto fahrendes Mitglied nass. (NZZ 43/2003)

Die Gründe für die auffällige Bedeutungsaktualisierung können in der bloßen Freude an der Sprache, in der Lust zur Kreativität im Gebrauch der sprachlichen Einheiten und vor allem im Interesse des Erreichens eines bestimmten wortspielerischen Überraschungseffekts liegen. Neben Steigerung der Bildhaftigkeit, der Expressivität und der Dynamik, die ein Phraseologismus im kreativen Gebrauch in den Text einbringt, streben die phraseologischen Auffälligkeiten durch formale oder semantische Aktualisierungen weitere unterschiedliche Wirkungen an. Sie können Träger einer wichtigen Information sein, die durch die gezielte 'auffällige' Benennung erreicht wird. ${ }^{29}$ Einige der wortspielerischen Funktionen, die Schifko (1987: 71) nennt, sind Amüsement, Heiterkeit, Komik über Persuasion, Manipulation, Ästhetik bis hin zu Verfremdung, Ironie und Spott sowie Sprach- und Gesellschaftskritik. Auch der in den Massenmedien angestrebte Leseanreiz wird durch auffällige phraseologische Aktualisierungen verstärkt. Kreative Texte zeugen von der Sprachkompetenz des Journalisten und setzen diese auch beim Leser voraus. Erst wenn diese bei beiden vorhanden ist, entsteht ein Sprachraum für die spielerische Verwendung von Phraseologismen. Das Produkt der Aktualisierung ist eine okkasionelle, einmalige Prägung, die nach einer Dekodierung und Interpretation durch den Rezipienten verlangt (vgl. Piirainen 1999: 263f.).

Kreative phraseologische Auffälligkeiten in der Funktion des Leseanreizes und der Weckung der Aufmerksamkeit sind für den Titelbereich charakteristisch (vgl. Pociask 2007b: 628).

(38) Na dobre i coraz lepsze (Polityka 3/ 2003)

(39) O trzech takich co... (GW 56/2011)

(40) Polak, Ukrainiec dwa bratanki (Wprost 9/2014)

Das Ziel der Zeitungsüberschriften besteht darin, die Aufmerksamkeit eines oft flüchtig und selektiv lesenden Rezipienten zu gewinnen, ihn anzusprechen und auf den anschließenden Haupttext zu leiten. Je nach Medium, thematischem Bereich und Zielgruppe bedient sich die Schlagzeile unterschiedlicher sprachlicher und stilistischer Mittel. Auffällige phraseologische

\footnotetext{
${ }^{27}$ Der Artikel handelt von verschiedenen Gefahren beim Erklimmen von Gipfeln. In demselben Text findet sich noch eine Demotivierung: Beispiel (30): To tylko wierzchotek góry lodowej.

${ }^{28}$ Das Spiel ergibt sich oft aus der kontextuellen Verwendung des usualisierten Phraseologismus, die jedoch im gegebenen Kontext fragwürdig, überraschend oder auffallend und dadurch amüsant und komisch wirkt. Es handelt sich um eine überraschende inhaltliche und formale Verknüpfung bei formal usuell oder auffällig aktualisierten Phraseologismen.

${ }^{29}$ Majkowska (1996: 40) spricht unter anderem von der nominativen Funktion von Phraseologismen in Pressetexten. Diese Funktion tritt oft in Verbindung mit der persuasiven Funktion auf.
} 
Aktualisierungen können diese Aufgabe der Schlagzeile erfüllen. Steht ein Phraseologismus im Titelbereich, so sichert ihm schon die herausgehobene Stellung die intendierte Beachtung (vgl. Błachut 2007: 53). Er wirkt interesseanreizend und lesestimulierend und kann bei einer entsprechenden Aktualisierung die Funktion der "zusammenfassend interpretierendwertenden Vorwegnahme des Hauptinhalts eines Beitrags" (Koller 1975: 400) übernehmen, sodass er zusätzlich die Aussagekraft der Schlagzeile unterstützt und als Aufmerksamkeitserreger und Spannungserzeuger fungiert.

Burger (1987a: 20) sieht einen weiteren Grund für die kreative metasprachliche Tätigkeit, die im Rahmen des Sprachspiels geschieht, darin, dass
"ein gekonnter, bis zu einem gewissen Grade auch 'kreativer' Umgang mit Phraseologie zu den professionellen Anforderungen an einen Journalisten gehört. Und hier bietet sich dem einzelnen Journalisten die Möglichkeit, jenseits der Kurzlebigkeit der journalistischen Wegwerfprodukte etwas wie Individualstil mit Anspruch auf sprachliche End-Gültigkeit zu entwickeln".

\section{Funktionen von Phraseologismen}

Bei der Bestimmung von Funktionen muss generell zwischen drei möglichen Funktionstypen unterschieden werden. Erstens soll zwischen textsemantischen und -konstitutiven Funktionen, den generellen Funktionen von Phraseologismen im Text und Funktionen von Phraseologismen-In-Textfunktion unterschieden werden.

Im Laufe der angestellten Überlegungen wurden bereits viele textkonstitutive und semantische Funktionen und generelle Funktionen genannt. Błachut (2007: 49-56) nennt und belegt am phraseologischen Material weitere semantische Effekte/Funktionen modifizierter formelhafter Wendungen und textstrukturierende Aufgaben. Zu nennen wären hier zum einen die Nuancierung der Bedeutung, Ironisierung, Distanzierung, der Motivierungseffekt und zum anderen die Einführung in das Thema und Pointierung ${ }^{30}$. Hervorzuheben sei, dass sich diese Funktionen aus den auffälligen formalen Aktualisierungsmöglichkeiten und der Positionierung der Phraseologismen im Text ergeben. Dabei kann kein Anspruch auf Vollständigkeit aller semantischer Effekte/Funktionen im Text erhoben werden, weil die Realisierung der Funktion in vieler Hinsicht von wendungsinternen und -externen Elemente bei der textuellen Aktualisierung und Art der formalen Veränderung sowie vom Aufkommen von Phraseologismen in jeweiligen Textteilen abhängt.

Neben den bereits erwähnten generellen Funktionen von Phraseologismen lassen sich weitere nennen. ${ }^{31}$ Ergänzend soll auf Fleischer (1997: 25) verwiesen werden, der unter dem kommunikativ-pragmatischen Aspekt und bezüglich der pragmatischen Potenz die folgende Eigenschaft von Phraseologismen hervorhebt:

"Eine wesentliche Rolle spielt die Fähigkeit der Phraseologismen, "psychische Zustände" des Sprechers oder Schreibers zu indizieren und beim Hörer oder Leser zu induzieren. Sie können dem Wecken oder Wachhalten der Aufmerksamkeit des Hörers oder Lesers dienen, der Herstellung engeren Kontaktes, der Betonung sozialer Zusammengehörigkeit, dem Ansprechen des Partners durch Bezugnahme auf seine Alltagserfahrung mit der Verwendung alltäglicher Formeln, auch der emotional betonten Wertung".

\footnotetext{
30 Vgl. dazu die Konklusionspointierungsfunktion bzw. Zusammenfassungsfunktion bei Sandig (1989: 394).

${ }^{31}$ Vgl. dazu die generellen stilistischen Funktionen von Sandig (1989).
} 
Auch Regers (1977: 344) Feststellung bezieht sich auf generelle Funktionen von Phraseologismen in Texten: "Die Journalisten benutzen die satirischen, ironischen und humorvollen Redewendungen, um bildhaft-kräftig und z.T. engagiert Stellung zu nehmen und Kritik zu üben. Die Idiome wirken lesemotivierend und fordern Zustimmung oder Gegenpositionen heraus". Den meisten Phraseologismen ist gemeinsam, dass sie die gereihten Informationen auflockern, indem sie der berichtenden Sprache im Pressentexten Bildhaftigkeit und Expressivität verleihen (vgl. Reger 1977: 342). Durch die Einführung von sprachlicher Bildlichkeit in sachlich informierenden Aussagen entsteht auch emotionalisierende Wirkung. ${ }^{32}$ So können Phraseologismen in informationsbetonten wie auch in meinungsbetonten Pressetexten auftreten und zur Verdeutlichung der Argumentation und der Pointe sowie als emotional wertende Darstellung von Sachverhalten eingesetzt werden (vgl. Sandig 1989: 392).

Fasst man im Zusammenhang mit der Bestimmung der Funktionen den textkonstitutiven Aspekt ins Auge, so muss gesagt werden, dass Phraseologismen als textsortenspezifische sprachliche Mittel an der Textkonstitution teilnehmen und die Realisierung der Textfunktionen unterstützen. ${ }^{33}$ Damit wäre der dritte Funktionstyp angesprochen. Die Aussagen über Funktionen der Phraseologismen lassen sich jedoch m. E. nicht immer verallgemeinern. Problematisch erscheint nämlich, Phraseologismen und ihre Funktionen losgelöst zu betrachten, denn die Funktion eines Phraseologismus sollte mit der Funktion des Textes, in dem er gebraucht wird, 'kompatibel' sein. Daher muss immer von potenziellen Funktionen gesprochen werden. Dies aus dem Grund, weil die Funktion des Phraseologismus immer als Funktion-im-Text gesehen werden muss (vgl. Pociask 2007a: 129). Außerdem kann ein Phraseologismus je nach Text die Textfunktion verstärken, sie ändern, teilweise oder ganz aufheben. Um seine Funktion-In-Textfunktion zu diskutieren, müssen die Funktionen der Korpustexte ins Auge gefasst werden.

Bei der Bestimmung der Textfunktion gehe ich von der Theorie aus, dass Texte kommunikative Größen und mit kommunikativen Funktionen versehen sind. Die Textfunktion stellt eine durch die "konventionell geltenden, in der Kommunikationsgemeinschaft verbindlich festgelegten Mittel ausgedrückte Kommunikationsabsicht des Emittenten" dar (Brinker 2005: 95). Es handelt sich also um eine bestimmte Absicht des Textproduzenten, die der Textrezipient erkennen soll. Sie stellt eine Anweisung des Produzenten an den Rezipienten dar, wie er den Text insgesamt auffassen

\footnotetext{
32 In diesem Zusammenhang ist auf die Vermischung von journalistischen Textsorten zu verweisen. Im Bereich der Informationstexte gibt es eine Reihe von Textsorten, die ein übergreifendes Merkmal und eine Funktion aufweisen. Ihre Hauptfunktion ist in erster Linie zu informieren. Sie unterscheiden sich jeweils in der Zusatzfunktion und ihrer intentionalen Ausrichtung der Vermittlung von Informationen (informationsbetont und meinungsbetont). Diese Tendenz zur Vermischung von Textsorten, so Burger (2005: 224), "ergibt sich aus der Tatsache, dass in allen Medien heute nicht mehr klar zu unterscheiden ist zwischen "informationsbetonten" und "meinungsbetonten" Texten, insbesondere zwischen Bericht und Kommentar". Journalisten versuchen neben Informieren auch Wünsche und Bedürfnisse sowie das Leseverhalten der Menschen zu berücksichtigen und ihre Meinung zu beeinflussen. Daher gilt auch für reine informationsbetonte Pressetexte, den Leseanreiz zu erhöhen. Texte, die kein Interesse wecken und keine Aufmerksamkeit auf sich ziehen, wirken nicht lesenswert. Die lesewerbende Wirkung kann unter anderem durch die Verwendung von Phraseologismen erreicht werden.

33 Es ist z. B. nicht zu leugnen, dass derselbe Phraseologismus z. B. in einem Werbetext die appellative Funktion und in einem Fachtext die Informationsfunktion realisieren bzw. unterstützen.
} 
soll, z. B. als informativ oder appellativ. Ich knüpfe in diesem Zusammenhang an Brinker (2005) an und zitiere seine Definition der Textfunktion. Er fasst die Textfunktion auf als "den Sinn, den ein Text in einem Kommunikationsprozess erhält, bzw. als einen Zweck, den ein Text im Rahmen einer Kommunikationssituation erfüllt" (Brinker 2005: 84). Unter dem kommunikativ-funktionalen Aspekt der interpersonalen Beziehung wird zwischen Informationsfunktion, Appellfunktion, Obligationsfunktion, Kontaktfunktion und Deklarationsfunktion unterschieden (vgl. Brinker 2005: 107). Demnach werden nach Brinker (2005: 135) fünf Textklassen unterschieden: Informationstexte, Appelltexte, Obligationstexte, Kontakttexte und Deklarationstexte. Daraus ist leicht ersichtlich, welche Grundfunktionen die Texte haben können. Weil die Korpustexte, aus denen Phraseologismen exzerpiert wurden, Informations- und Appelltexte (Meldungen, Berichte, Reportagen und Kommentare, Glossen) sind und somit die Informations- und Appellfunktion erfüllen, sollen auf diese kurz eingegangen werden.

Mit der Informationsfunktion versucht der Textproduzent dem Rezipienten sein Wissen zu vermitteln, mit der Absicht, ihn zu informieren. Charakteristisch ist die Informationsfunktion für Textsorten wie Nachrichten, Berichte und Sachtexte. "Die informative Textfunktion kann sich mit der evaluativen Einstellung des Sachverhalts verbinden" (Brinker 2005: 109). Mit der Information kann der Textproduzent sekundär seine (positive oder negative) Bewertung ausdrücken.

Die Appellfunktion liegt dann vor, wenn der Textproduzent mit seinem Text den Rezipienten zur Meinungs- und/oder Verhaltensänderung bewegen bzw. die Meinungsbildung und das Verhalten des Rezipienten beeinflussen möchte. Sie ist charakteristisch u.a. für die Pressetextsorten wie Kommentare und Glossen. Auch Lüger (1995) befasst sich mit der Funktion von Pressetexten und nennt dabei folgende Textklassen: meinungsbetonte Texte (Lüger 1995: 125-144), auffordernde Texte (Lüger 1995: 144-147) und instruierendanweisende Texte (Lüger 1995: 147-151). In der letzten Textklasse differenziert er zwischen Handlungsanleitungen und Ratgebungen. Die meinungsbetonten Texte bei Lüger beziehen sich auf die Meinungsbeeinflussung und die instruierend-anweisenden auf die Verhaltenssteuerung. $\mathrm{Zu}$ problematisieren ist die bereits angesprochene allgemeine Tendenz der Vermischung von journalistischen Texten (vgl. Majkowska 2001: 25), was dazu führt, dass auch Informationstexte meinungsbildend und -beeinflussend sein können bzw. die Grenze zwischen Informieren und Beeinflussen nicht selten verwischt wird, denn die Darstellungsform gelieferter Informationen kann - je nach Verwendung bestimmter sprachlicher Formen/Strukturen im Text unmittelbar oder innertextlicher und außertextlicher Merkmale mittelbar - "zur Verbesserung oder Vermeidung eines als für den Adressaten negativ, defizitär oder problematisch beurteilten Zustands beitragen" (Lüger 1995: 147) und sich zwischen den Polen informativ - meinungsbetont bewegen.

Im Zusammenhang mit der Textfunktion ist nach der 'Funktion der Phraseologismen-InTextfunktion' zu fragen. Der Textproduzent bedient sich bei der Gestaltung des Textes fester Phrasen, um bestimmte Textfunktionen zu realisieren. Das bedeutet, dass Phraseologismen als innersprachliche Mittel einen Anteil an der Realisierung der Textfunktion haben. Realisiert werden also diejenigen Funktionen von Phraseologismen, die in Texten die Informationsbzw. Appellfunktion unterstützen. Kollers (1977) Arbeit, in der er die Funktionen von Redewendungen ausführlich untersucht, ist umso interessanter, als ihr Schwerpunkt in der 

dargestellt aus pragmalinguistischer Perspektive

Untersuchung von Zeitungstexten liegt, die deutlich machen, welche Funktionen und Wirkung Phraseologismen im Text haben können. Koller (1977: 142-145) differenziert zwischen der Funktion der Vereinfachung, Argumentationsersparung, Anbiederung, Unschärfe, Anschaulichkeit und der Übertragung.

- Vereinfachungsfunktion - Phraseologismen vereinfachen schwer durchschaubare Zusammenhänge, ohne dabei den Sachverhalt selbst zu reduzieren.

- Funktion der Argumentationsersparung ${ }^{34}$ - Phraseologismen dienen als verkürzte Argumentation. Verstärkt wird die verkürzte Argumentation durch die einigen festen Phrasen eigens zugeschriebene Bildhaftigkeit und Expressivität.

- Anbiederungsfunktion - Mit der Verwendung von Phraseologismen signalisiert der Textproduzent dem Rezipienten, dass er fähig ist, die gegebene Situation zu erfassen und zu beurteilen.

- Unschärfefunktion - Phraseologismen wirken auf den ersten Blick zwar einleuchtend, sind dann aber oft sehr verallgemeinernd und unklar.

- Anschaulichkeitsfunktion - Phraseologismen eigenen sich zur anschaulichen und bildlichen Darstellung komplexer Sachverhalte und zur Erläuterung eines komplizierten Zusammenhangs.

- Übertragungsfunktion - Phraseologismen veranschaulichen komplizierte Zusammenhänge der gegebenen Situation verständlich, indem sie sie in einfache Zusammenhänge des Alltagslebens überführen.

Die letzten zwei Funktionen können als Vereinfachungsfunktion aufgefasst werden, da Phraseologismen durch Veranschaulichung und Übertragung zum Verständnis komplizierter Sachverhalte beitragen.

Diese Funktionen werden von Koller als manipulativ eingestuft, weil sie die manipulativen Funktionen seiner Korpustexte realisieren. Es handelt sich dabei jedoch im Allgemeinen um ihre potenziell manipulativen Funktionen-In-Textfunktion. Zwar wurden diese Funktionen auf Grund der Untersuchung an konkreten Texten als manipulativ nachgewiesen, aber dieselben phraseologischen Einheiten können in anderen thematischen Bereichen, Textsorten oder Medien andere Funktionen-In-Textfunktion unterstützen bzw. realisieren. Daher können solche Funktionen nur am Beispiel konkreter Texte in einem bestimmten Textumfeld (Funktion, Kontext, Medium, Rezipientengruppe) bestimmt und nachgewiesen werden. Weil die Funktionen zur Unterstützung der Textfunktionen eingesetzt werden, kann von Unterstützungsfunktionen gesprochen werden, weil der Text nicht entsteht, um die Funktion von Phraseologismen zu demonstrieren, sondern die Phraseologismen werden als Mittel zur Unterstützung der Textfunktion eingesetzt. Selbstverständlich manifestieren die Einheiten ihre Funktionen im Text, sie dienen aber zur Realisierung der Funktion größerer Einheiten, nämlich der Texte.

\footnotetext{
34 Burger (1987a: 15) betont jedoch, dass Phraseologismen keine Argumentation an sich ersetzen können. Sie bilden lediglich einen Rahmen für Argumentation und können die argumentativen Formulierungen expressiv und bildlich unterstützen. Vgl. dazu auch Wirrer (2007): Phraseme in der Argumentation.
} 
Versucht man nun die phraseologischen Funktionen den Textfunktionen zuzuschreiben, so ergibt sich die folgende Zusammenstellung der Funktionen. Die Informationsfunktion der Meldungen und Berichte kann durch die Funktion der Vereinfachung, der Anbiederung oder der Anschaulichkeit unterstützt werden. Der Textproduzent informiert über schwer durchschaubare Zusammenhänge, indem er diese vereinfachend veranschaulicht.

Die meinungsbildende Funktion der Kommentare oder Glossen wird durch die Funktion der Argumentationsersparung oder die Unschärfefunktion realisiert. ${ }^{35}$ Der Textproduzent zwingt den Leser zur Meinungsbildung durch Argumentation oder "unscharfe" expressive Formulierungen, die zum Nachdenken zwingen.

Wie man dem oben Angeführten entnehmen kann, sind Phraseologismen mit ihren Funktionen den nicht konkreten Textfunktionen fest zugeschrieben und können sogar entgegengesetzte Funktionen realisieren.

\section{$7 \quad$ Zur Position der Phraseologismen im Text}

Grundsätzlich können Phraseologismen an jeder Position im Text erscheinen, allerdings mit unterschiedlicher Quantität und in unterschiedlicher formaler Ausprägung. Die meisten kommen im Haupttext (84\%) vor, im Lead (1,5\%) und im Titelbereich (14,5\%). In diesem Zusammenhang soll nach dem funktionalen Aspekt gefragt werden. Betrachtet man die Form der Phraseologismen in den Positionen im Text, so muss festgehalten werden, dass sich auffällige Aktualisierungen besonders im Titelbereich großer Beliebtheit erfreuen. Für das kreative Umgehen im Titelbereich eignen sich Phraseologismen aus zweierlei Gründen: Es ist zum einen ihre hohe Expressivität, die durch die auffällige Form (z. B. Reduktion) verstärkt wird. Zum anderen übernehmen sie die Funktion 'eines sprachlichen Bildes', das hinter dem ihnen steht und als Leseanreiz dient. "Bei Verkürzungen auf den unikalen nominalen Teil kann der konkrete Bezug zunächst in der Schwebe bleiben und erst durch den späteren Kontext erhellt werden" (Wotjak 1992: 147). Solche Reduktionen werden oft beim kreativwortspielerischen Umgang mit Phraseologismen genutzt. Gerade für den Text hat das Weglassen von phraseologischen Komponenten bei gleichzeitiger Erweiterung um für den Text relevante Elemente in der Schlagzeile eine wesentliche Bedeutung. Die fehlenden Teile werden ohne Schwierigkeiten ausgefüllt, weil sie, wie Schweizer (1978: 17) sagt, auf der Hand liegen.

Wenn Phraseologismen im Titelbereich und an unterschiedlichen Stellen im Haupttext vorkommen, so haben sie ohne Zweifel eine textorganisierende Funktion. In diesem Zusammenhang soll auf die Feststellung von Skog-Södersved (2007: 272) hingewiesen werden, dass die Schlagzeile und der Haupttext bei vielen journalistischen Texten nicht von derselben Person stammen. ${ }^{36}$ Daher wird hier behauptet, dass Phraseologismen in der

\footnotetext{
35 Die den Phraseologismen eigene semantische Unschärfe, Dehnbarkeit und ihre Vagheit (Burger 1998: 77-78) gewährleisten die Anpassungsfähigkeit an verschiedene Kontexte und ermöglichen die Realisierung unterschiedlicher Funktionen im Text. Die textsemantische Unschärfe und pragmatische Vagheit wird durch die Lektüre des Textes beseitigt.

${ }^{36}$ Interessant scheint in solchen Fällen die Frage nach der Abfolge der Textproduktion. Wird der Titel zum Haupttext formuliert oder der Haupttext zum (Arbeits)Titel? Zu vermuten ist, dass es Unterschiede zwischen den Darstellungsformen gibt.
} 
Schlagzeile in gewissem Sinne der Ausgangspunkt für Wiederaufnahmen, Rückverweise und Wiederholungen eines ganzen Phraseologismus oder seiner Komponenten sind. Sie bilden sozusagen die Orientierung und zugleich das Gerüst für den Inhalt und Textverlauf. Wenn man annimmt, dass sich die Textproduzenten bzw. 'Titelverfasser' am Textinhalt orientieren, so erfüllen Phraseologismen in der Schlagzeile die bereits von Koller (1975: 400) erwähnte Funktion der "zusammenfassend interpretierend-wertenden Vorwegnahme des Hauptinhalts eines Beitrags". Am Ende des Textes dagegen fungieren sie nicht selten als ein pointierter Textschluss (vgl. Skog-Södersved 2007: 272). Daher kommt ihnen als solchen eine besondere Rolle für die Textgestaltung zu.

\section{$8 \quad$ Zusammenfassung}

Auf Grund der durchgeführten Analyse und ihrer quantitativen Ergebnisse ist festzustellen, dass Phraseologismen als stilistische und textkonstitutive Mittel ein fester sprachlicher Bestandteil der Pressetexte sind. Behauptet wird dies auf Grund ihres häufigen Erscheinens in den Texten und äußert sich besonders in ihrer textbildenden Funktion. Ihr Beitrag zur Textstruktur und zum Textinhalt zeigt sich in der Gewährleistung der Textkonstitution.

Vor allem in kommentierenden Pressetexten, die zwar tatsachenbetont, aber auch persönlich gefärbt berichten, bieten sich Möglichkeiten zum kreativen Jonglieren mit der Sprache, unter anderem zur auffälligen Aktualisierung, die einerseits an der formalen Ebene und andererseits an der Semantik der Phraseologismen ansetzten. Diese Techniken sind oft in den meinungsbetonten und vereinzelt in den informierenden Darstellungsformen in der Presse zu finden. Phraseologismen, an der rechten Stelle im Text und auffällig eingebettet, stellen in vieler Hinsicht einen Mehrwert für den Text dar. Der Mehrwert der auffälligen Aktualisierungen besteht in einem bestimmten stilistisch-semantischen und kommunikativpragmatischen Effekt: Der Leser soll angesprochen, der Leseanreiz erhöht werden. Bei kreativen Aktualisierungen unterschiedlicher Ausprägung handelt es sich um bewusst vorgenommene pragmatische Veränderungen, bei denen die Identität des Phraseologismus nicht beeinträchtigt wird (vgl. Černyševa 1984: 20), sondern zum Ausdruck kommt, denn gerade die Variabilität, die Phraseologismen kontextuell zulassen, stellt eines ihrer charakterisierenden Merkmale dar.

Das wichtigste Erkenntnisinteresse ist aus der pragmatischen Perspektive auf Funktionen gerichtet. Unter dem pragmatisch orientierten Aspekt muss davon ausgegangen werden, dass ein Phraseologismus ein bestimmtes Spektrum von Funktionen erfüllen kann. Diese Polyfunktionalität ist jedem Phraseologismus zugeschrieben. Aus dieser Eigenschaft heraus ergibt sich die Tatsache, dass man erstens nur von ihren potenziellen textsemantischen und textbildenden Funktionen sprechen kann, weil nur im konkreten Text durch eine entsprechende Aktualisierung ersichtlich wird, welche der potenziellen Funktionen realisiert wird/ werden. Zweitens können Phraseologismen generelle Funktionen im Text erfüllen. Diese Funktionen ergeben sich aus ihrer semantischen Beschaffenheit. Im Bestreben nach interessanter Textgestaltung, wirkungsvoller Ansprache und kreativer journalistischer Individualität bedienen sich Textproduzenten unterschiedlicher Gestaltungsmethoden und mittel. Phraseologismen repräsentieren größtenteils bildhafte Ausdrucksweisen und durch ihre Verwendung gewinnen Pressetexte an Lebendigkeit und sprachlicher Attraktivität. Sie eignen sich besonders für eine charakteristische stilistische Verwendung, für Selbstdarstellung und 
den Ausdruck emotional bewertender Einstellungen. Drittens ist aus der pragmalinguistischen Perspektive interessant und notwendig, Funktionen von Phraseologismen-In-Textfunktion zu analysieren. Dies ist dadurch begründet, dass ihre Funktionen Bestandteile übergeordneter Textfunktionen sind und im Dienste der Realisierung der Textfunktionen stehen.

Vergleicht man die Häufigkeit des Vorkommens in informationsbetonten und meinungsbildenden Texten, so ist festzustellen, dass meinungsbildende Kommentare und Glossen (ca. 91\%) wesentlich mehr Phraseologismen enthalten als informierende Texte wie Nachrichten, Berichte und Meldungen (ca. 9\%). Interessant ist jedoch ihre formale Ausprägung im Text: In Kommentaren und Glossen treten sie gehäuft, abgewandelt und kontaminiert auf. In informationsbetonten Texten dagegen, abgesehen von einigen Erweiterungen um Komponenten, die neue Informationen in den Text einbringen, erscheinen sie in ihrer usuellen Form. Auch in einigen Meldungen finden sich Phraseologismen im Titelbereich. ${ }^{37}$ Hier aktivieren sie Leerstellen, die mit Information verdichtet werden und realisieren die Informationsfunktion der Texte. Durch die Verwendung von erweiterten Phraseologismen kann in sachlich informierenden Aussagen auch emotionalisierende Wirkung entstehen. ${ }^{38}$

Unterschiede sind jedoch in der Realisierung und Aktivierung der Leerstellen im Komponentenbestand in verschiedenen Texten und dadurch in textsemantischen Funktionen festzustellen. In meinungsbetonten Texten werden meistens Funktionen der Konkretisierung und Spezifizierung sowie der Intensivierung und Emotionalisierung der Ausdrucksweise realisiert. Dagegen hat der 'Eingriff' in die Struktur des Phraseologismus in den informierenden Texten die Aufgabe der Informationsverdichtung und Nomination.

Es ist ferner festzuhalten, dass Phraseologismen in ihren generellen Funktionen im Text (Anschaulichkeit, Expressivität, Pointierung) in informationsbetonten Pressetexte nicht eingesetzt werden. Darüber hinaus sind inhaltliche Auffälligkeiten und sprachspielerische Effekte wie Amüsement, Heiterkeit, Komik und Persuasion ausschließlich in meinungsbildenden Texten vorzufinden.

Es sei hier hervorgehoben, dass die auffälligen formalen und die vom Kontext gesteuerten inhaltlichen Aktualisierungen der Phraseologismen sowie die textuelle Realisierung der Funktionen auf Grund der Aktivierung ihrer textbildenden Potenzen möglich sind.

Die Untersuchungen zeigen, dass hinsichtlich der Quantität der auffälligen Aktualisierungen im Text erhebliche Unterschiede bestehen. Der Anteil der formalen Auffälligkeiten beträgt in Pressetexten bis zu 50\%. Meine Untersuchungen bestätigen eine steigende Tendenz. ${ }^{39}$ Einerseits kann der Grund der ansteigenden Tendenz darin liegen, dass man im Bestreben nach kreativer Ausdrucksweise bekannte Phraseologismen nicht einfach wiederholt. In

\footnotetext{
${ }^{37} \mathrm{Zu}$ erklären ist dies aus der bereits erwähnten Tendenz zur Vermischung von Pressetextsorten. Vgl. dazu die Untersuchung von Misiek (2011: 129), in der sie behauptet: "Für Phraseolexeme, die eine Einstellung des Emittenten mitvermitteln, bleibt somit in den Meldungen kein Platz".

38 Dazu Beispiele: (41) Fettnäpfchen (SZ vom 23.11.2012), (42) Im Visier der NATO (Der Spiegel 50/2013), (43) Na garnuszku Moskwy (Wprost 14/2014).

39 Nach Černyševa (1980) beträgt der Anteil der Modifikationen in publizistischen Texten bis zu 30\%. Wotjak (1992) spricht von $35 \%$ bis $50 \%$ und Pociask (2007a) von $37 \%$.
} 
Pressetexten kommen aus diesem Grund verschiedene Varianten, Variationen und auffällige formale und semantische Aktualisierungen der Phraseologismen vor. ${ }^{40}$

Abschließend soll festgehalten werden, dass es kaum Unterschiede in den Pressetexten zwischen beiden Sprachen gibt. Dies betrifft sowohl formale und inhaltliche Aktualisierungen, Häufigkeiten der Verwendung und Funktionen. Hier wird davon ausgegangen, dass es sich bei Phraseologismen um sprachliche Universalien handelt und dass feste Phrasen nicht nur im Sprachsystem als solche fungieren, sondern auch als sprachliche textsortenspezifische Textbausteine in vielen Sprachen ähnliche textkonstitutive und textsemantische Funktionen übernehmen und ihre pragmatischen Potenzen im Gebrauch entfalten.

\section{Bibliographie}

Barz, Irmhild (1986): "Probleme der phraseologischen Modifikation". Deutsch als Fremdsprache. Zeitschrift zur Theorie und Praxis des Deutschunterrichts für Ausländer 23/1986: 321-326.

Bąba, Stanisław (1989): Innowacje frazeologiczne współczesnej polszczyzny. Poznań: Wydawnictwo Naukowe UAM.

Błachut, Edyta (2004): Sprachspielerische Modifikationen formelhafter Wendungen. Untersuchungen anhand deutscher und polnischer Belege. Wrocław: ATUT.

Błachut, Edyta (2007): "Textsemantische Funktionen modifizierter formelhafter Wendungen". In: Cirko, Lesław/Grimberg, Martin (eds.) (2007): Sprachlust - Norm - Kreativität. Wrocław, ATUT: 47-58.

Brinker, Karl (2005): Linguistische Textanalyse. Eine Einführung in Grundbegriffe und Methoden. 6. Auflage. Berlin: Erich Schmidt.

Burger, Harald/Buhofer, Annelies/Sialm, Ambros (1982): Handbuch der Phraseologie. Berlin/New York: de Gruyter.

Burger, Harald (1987a): "Funktionen von Phraseologismen in den Massenmedien". In: Burger, Harald/Zett, Robert (eds.) (1987): Aktuelle Probleme der Phraseologie. Frankfurt a. M. etc., Lang: 11-29. (= Zürcher Germanistische Studien 9)

Burger, Harald (1987b): "Normative Aspekte der Phraseologie". In: Korhonen, Jarmo (ed.) (1987): Beiträge zur allgemeinen und germanistischen Phraseologieforschung. Oulu: 6590. (=Veröffentlichungen des Germanistischen Instituts 7)

Burger, Harald (1998): Phraseologie: Eine Einführung am Beispiel des Deutschen. Berlin: Schmidt.

Burger, Harald (2005): Mediensprache: eine Einführung in Sprache und Kommunikationsformen der Massenmedien. Berlin: de Gruyter.

Burger, Harald et al. (eds.) (2007): Phraseologie/Phraseology. Ein internationales Handbuch der zeitgenössischen Forschung. Berlin/New York: Walter de Gruyter.

Černyševa, Irina (1980): Variabilität in Sprachsystem, und Text auf lexikalischphraseologischer Ebene. Zeitschrift für Phonetik, Sprachwissenschaft und Kommunikationsforschung 33: 307-310.

\footnotetext{
${ }^{40}$ In diesem Zusammenhang diskutiert Mosiołek-Kłosinska (2002) Mechanismen phraseologischer Innovationen als Quelle für die Entstehung neuer expressiver Benennungen.
} 
Černyševa, Irina (1984): "Aktuelle Probleme der deutschen Phraseologie". Deutsch als Fremdsprache. Zeitschrift zur Theorie und Praxis des Deutschunterrichts für Ausländer. 21/1984: 17-22.

Dobrovol'skij, Dimitrij (1980): "Zur Dialektik des Begriffs der textbildenden Potenzen von Phraseologismen". Zeitschrift für Phonetik, Sprachwissenschaft und Kommunikationsforschung 33: 690-700.

Donalies, Elke (2012): Phraseologie. Studienbibliographien Sprachwissenschaft 40. Tübingen: Narr.

Elspaß, Stephan (2002): "Alles unter den Tisch gekehrt - Phraseologische Gebrauchsauffälligkeiten im Urteil von Sprachbenutzern". In: Hartmann, Dietrich/Wirrer, Jan (eds.) (2002): "Wer A sägt, muss auch B sägen." Beiträge zur Phraseologie und Sprichwortforschung aus dem Westfälischen Arbeitskreis. Hohengehren, Schneider: 127160.

Filatkina, Natalia (2007): "Pragmatische Beschreibungsansätze". In: Burger, Harald et al. (eds.) (2007): Phraseologie/Phraseology. Ein internationales Handbuch der zeitgenössischen Forschung. Berlin/New York, Walter de Gruyter: 132-158.

Fleischer, Wolfgang ( $\left.{ }^{2} 1997\right):$ Phraseologie der deutschen Gegenwartssprache. Tübingen: Niemeyer.

Fliciński, Piotr (2011): Innowacje frazeologiczne $w$ świetle danych korpusowych. Poznańskie Studia Polonistyczne: 9-19. (= Linguistic Series 18/2011)

Földes, Csaba (1997): Idiomatik/ Phraseologie. Heidelberg: J. Groos. (= . Studienbibliographien Sprachwissenschaft 18)

Gréciano, Gertrud (1983): "Forschungen zur Phraseologie". Zeitschrift für germanistische Linguistik 11: 232-243.

Gréciano, Gertrud (1987): "Idiom und Text". Deutsche Sprache 15: 193-208.

Hessky, Regina (1992): "Grundfragen der Phraseologie". In: Ágel, Vilmos/Hessky, Regina (eds.) (1992): Offene Frage, offene Antworten in der Sprachgermanistik. Tübingen: Niemeyer: 77-93.

Koller, Werner (1975): "Redensarten in Schlagzeilen". Muttersprache 85/6: 400-408.

Koller, Werner(1977): Redensarten. Linguistische Aspekte, Vorkommensanalysen, Sprachspiel. Tübingen: Niemeyer.

Lewicki, Andrzej (2007): "Rozwój frazeologii polskiej w ostatnim ćwierćwieczu". In: Lewicki, Andrzej (ed.): Problemy frazeologii europejskiej VIII. Lublin, Norbertinum: 914.

Lüger, Heinz-Helmut (1995): Pressesprache. Tübingen: Niemeyer.

Majkowska, Grażyna (1996): "Funkcje frazeologizmów w tekstach publicystycznych". In: Lewicki, Andrzej (ed.) (1996): Problemy frazeologii europejskiej I. Lublin, Norbertinum: $39-47$.

Majkowska, Grażyna (2001): "Najnowsza frazeologie publicystyczna". In: Lewicki, Andrzej (ed.) (2001): Problemy frazeologii europejskiej IV. Lublin, Norbertinum: 25-33.

Misiek, Dorota (2011): "Phraseologismen in deutschen und polnischen Pressetexten". In: Schiewe, Jürgen et al. (eds.) (2011): Kommunikation für Europa II. Sprache und Identität. Frankfurt a. M. etc., Lang: 123-135. 
Mosiołek-Kłosińska, Katarzyna (2002): "Innowacje frazeologiczne jako źródło powstania nowych jednostek leksykalnych". In: Lewicki, Andrzej (ed.) (2002): Problemy frazeologii europejskiej $V$. Lublin, Norbertinum: 21-34.

Pajdzińska, Anna (2001): "O znaczeniu zwiazku frazeologicznego (raz jeszcze)". In: Lewicki, Andrzej (ed.) (2001): Problemy frazeologii europejskiej IV. Lublin, Norbertinum: 11-18.

Palm, Christine (1995): Phraseologie: eine Einführung. Tübingen: Narr.

Piirainen, Elisabeth (1999): "Das geht durch Mark und Pfennig. Usualisiertes Wortspiel in der deutschen Phraseologie". In: Baur, Rupprecht S./Chlosta, Christoph/Piirainen, Elisabeth (eds.) (1999): Wörter in Bildern. Bilder in Wörtern. Beiträge zur Phraseologie und Sprichwortforschung aus dem Westfälischen Arbeitskreis. Hohengehren, Schneider: 263-282.

Pociask, Janusz (2007a): Zu Status und Funktion der idiomatischen Einheit in Pressetexten. Frankfurt a. M. etc.: Lang.

Pociask, Janusz (2007b): "Zur Funktion der Phraseologismen in Zeitungsüberschriften". In: Kolago, Lech (ed.): Studia Niemcoznawcze XXXVI. Warszawa: 619-629.

Pociask, Janusz (2010): "Zur grammatischen Variabilität von Phraseologismen". In: Błachut, Edyta/Cirko, Lesław/Tworek, Artur (eds.) (2010): Studia Linguistica XXIX. Wrocław, Wydawnictwo Uniwersytetu Wrocławskiego: 93-102.

Reger, Harald (1977): "Zur Idiomatik der konventionellen Tagespresse". Muttersprache 87/5: 337-347.

Roos, Eckhart (2001): Idiom und Idiomatik. Ein sprachliches Phänomen im Lichte der kognitiven Linguistik und Gestalttheorie. Aachen: Shaker.

Sandig, Barbara (1989): "Stilistische Funktionen verbaler Idiome am Beispiel von Zeitungsglossen und anderen Verwendungen". In: Gréciano, Gertrud (ed.) (1989): EUROPHRAS 88. Phraséologie Contrastive. Actes du Colloque International Klingenthal Strasbourg. Strasbourg: 387-400.

Schifko, Peter (1987): "Sprachspiel und Didaktik der Linguistik". Zeitschrift für Romanische Philologie 103/1/2: 68-87.

Schweizer, Blanche-Marie (1978). Sprachspiel mit Idiomen: eine Untersuchung am Prosawerk von Günter Grass. Zürich: Druk.

Skog-Södersved, Mariann (2007): "Phraseologismen in den Printmedien". In: Phraseologie/Phraseology. Ein internationales Handbuch der zeitgenössischen Forschung. Berlin/New York, de Gruyter: 269-275.

Wirrer, Jan (2007): "Phraseme in der Argumentation". In: Burger, Harald et al. (eds.): Phraseologie/Phraseology. Ein internationales Handbuch der zeitgenössischen Forschung. Berlin/New York, de Gruyter: 175-187.

Wotjak, Barbara (1992): Verbale Phraseolexeme in System und Text. Tübingen: Niemeyer.

Wotjak, Barbara (1999): "Zu textuellen Vernetzungen von Phraseologismen am Beispiel von Sprachwitzen". In: Fernandez Bravo, Nicole/Behr, Irmtraud/Rozier, Claire (eds.): Phraseme und typisierte Rede. Tübingen, Niemeyer: 51-63. 